\title{
A Momentum-First Approach to Teaching Force in High School
}

\author{
Jarier Wannous ${ }^{a}$, Peter Horváth ${ }^{\text {b }}$ \\ ${ }^{a}$ Comenius University in Bratislava, Faculty of Mathematics, Physics and Informatics, Department of \\ Didactics in Mathematics, Physics and Informatics, jarier.wannous@fmph.uniba.sk, b Comenius \\ University in Bratislava, Faculty of Mathematics, Physics and Informatics, Department of Didactics \\ in Mathematics, Physics and Informatics, peter.horvath@fmph.uniba.sk
}

\begin{abstract}
A common conception that students have in mechanics courses is that a force is needed to maintain motion. Our work approaches this problem from a pprim perspective with a comparison to the historical understanding of force. From a p-prims perspective, momentum is proposed to substitute force as a maintaining agency to motion. By a comparison with history, it is shown that a similar problem occurred in the development of the concept of force, which led to a similar solution to the one proposed in our work. As a result, a teaching plan and activities are prepared to introduce the concept of force through the concept of momentum. In this work, the main activities of the plan are discussed, as well as the results of a preliminary research based on its realization in a classroom.
\end{abstract}

Keywords: momentum, force, teaching plan, history, p-prims.

\section{Introduction}

Since the first introduction of physics in history by Aristotle, understanding force and motion was the most problematic. However, that understanding was important for science, as the concept of force is one of the corner stones of physics. But just as this concept became a stumbling block for physicists in the past, it seems it became a problem for students today as well. McDermott (1997), for example, mentions many different studies that have dealt with students' understanding of force and motion. It seems that the most problematic and reoccurring preconception is that an impetus, an internal motive force, is needed to maintain motion.

In our paper, we focus on this preconception as we discuss it from two different viewpoints, the viewpoint of phenomenological primitives (p-prims) and the viewpoint of history. We 
believe those two viewpoints offer a significant advantage for the discussion about students' understanding of force and its effects on motion. Using the analysis of history and p-prims we offer a few recommendations for teachers to use in physics courses. The recommendations have been included in a teaching plan used over the course of the past few years in Slovak schools. A brief discussion of the effectiveness of the plan is also included in our paper.

\section{The Viewpoint of P-prims}

While preconceptions are understood to be "strongly held, stable cognitive structures; differ from expert conceptions; affect in a fundamental sense how students understand natural phenomena and scientific explanations; and must be overcome, avoided, or eliminated for students to achieve expert understanding" (Hammer, 1996), phenomenological primitives or p-prims can be understood as "intuitive knowledge that constitute people's 'sense of mechanism,' their sense of which happenings are obvious, which are plausible, which are implausible, and how one can explain or refute real or imagined possibilities" (diSessa, 2016). P-prims are not considered to be incorrect on their own. Their activation in certain contexts however is not desired.

In other words, while preconceptions are usually connected to a certain theory or a concept, p-prims are more basic and can be activated with different theories. For a clearer understanding of how p-prims work, we will show how they appear in the context of force and momentum. For these concepts, the main p-prims which we need to consider are:

- Maintaining agency: for a mechanism to function there must exist an agency that maintains that functionality, e.g. an engine maintains the motion of a car, or motion implies a force.

- Actuating agency: this p-prim simulates the law of causality. There must exist something that causes a certain effect, e.g. a hammer causes a bell to ring.

- Dynamic balance: a pair of effects that are equal in magnitude but in opposite directions balance and cancel each other.

- Overcoming: when one effect or influence overpowers another.

A more detailed list of p-prims can be found in Kortland's Handboek natuurkundedidaciek (Kortland \& Poorthuis, 2017).

In the context of force, students often encounter situations where maintaining motion requires a force. Such situations activate the maintaining agency p-prim and builds the preconception that force is proportional to velocity. However, unknown to the student is the 
fact that what he is experiencing is not a force working as a maintaining agency but as a dynamic balance to resistance. The true function of a force, if it is the only one affecting an object, is changing the momentum of that object.

That is why, we believe that in order to build a correct understanding of the role of force, it is important to introduce the true maintaining agency for motion before getting acquainted with the concept of force. We propose that the physical quantity which can be considered as a maintaining agency to motion is the momentum of the object. The main reason for this proposal is that conservation of momentum, although itself stemming from the role of force, is the law that keeps an object moving in case there are no external forces affecting it.

Only after the correct identification of the agency maintaining motion, the role of force can be truly identified as the actuating agency for motion, i.e. changing the momentum of the object. When the role of force is identified, a correct interpretation of previous experiences can be achieved through activating the dynamic balance and overcoming p-prims.

While a p-prims perspective offers much insight into the problem of understanding force, it is interesting that this perspective is paralleled in the way the concept of force developed in history, which in itself presents another positive argument why momentum should be introduced as the maintaining agency for motion.

\section{A Parallel in History}

To look at the historical development of the concept of force means to start with Aristotle and his book Physics. In it he mentioned a few ideas about force, of which the most important is that force is proportional to velocity or in his own words:

"Let the motive agency be $\alpha$, the moving body be $\beta$, the distance travelled $\gamma$ and the time taken by the displacement $\delta$. Then an equal power, namely power $\alpha$, will move half of $\beta$ along a path twice $\gamma$ in the same time, or it will move it through the distance $\gamma$ in half the time $\delta$. For in this way the proportions will be maintained" (Dugas, 1955).

This force was believed to be external as well as the cause of what Aristotle called, violent motion. As force according to Aristotle is always caused by an object in direct contact with the moving body (Baez, 2008), a definition of another type of motion, not caused by force thus defined, was needed. Aristotle therefore defined natural motion, as opposed to violent motion, as an object's tendency to move upwards to the heavens, which he called 'levity', or downwards to the center of the earth, which he called 'gravity' (Miao, 2016). Aristotle also indicated, when speaking of gravity, that the velocity of a falling object is directly 
proportional to its weight and indirectly proportional to the resistance of the medium (Baez, 2008).

One of the first people who criticized Aristotle's notions was John Philiponus of Alexandria in the 5th century. Philiponus laid the foundation for the theory of impetus, even though he never used the term in his work. His criticism was based on the fact, that Aristotle's mechanics gave a feeble explanation to the motion of a thrown projectile. Philiponus was one of the first who considered that the motion of a projectile after being released is due to a property of the object, while Aristotle claimed that the motive force is transferred to the air surrounding the projectile. Understanding that the medium does not maintain the motion of a projectile also lead Philiponus to grasp its true role in motion, which is resistance (Wildberg, 2016).

Philiponus' ideas were widely rejected until the 14th century, even though his work was known to many such as Albert Magnus and Thomas Aquinas. However, in the 14th century, his work became the basis of Jean Buridan's theory of Impetus (Hecht, 2015), which was formulated as following:

1. impetus is a kind of power, existing in the physical world, which is able to move objects in the same direction as the impetus itself,

2. impetus can be imparted to an object from another object,

3. depending on the amount of impetus imparted to an object, the object moves correspondingly to that amount,

4. impetus can be dissipated or weakened by the resistance in the medium,

5. because impetus can be either rectilinear or curvilinear, the motions produced by the impetus can also be straight or curved.

Even at first glance, we can notice parallels between the theory of impetus and momentum. The only exception is the 5th point, which uses impetus to explain motion on a curve. Buridan even proposed that impetus is the product of the amount of matter and speed (Graney, 2013) which can lead us to the following formula to calculate impetus:

$$
I=m v
$$

The theory of impetus became the stepping stone for defining momentum and its role in motion. Some, as was Albert of Saxony, used impetus to explain why a falling object does not infinitely increase its velocity but reaches a certain limit (Dugas, 1955). While others, like Galileo and Huygens, built upon the theory to reach the first correct understanding of inertia. In Galileo's Discorsi, for example, we can read the following: "I imagine a moving body thrown on a horizontal plane without any obstacle. It is said that its motion on the plane will remain uniform indefinitely if the plane extends to infinity" (Dugas, 1955). 
Reaching a correct understanding of momentum, through the previous theory of impetus, finally led to understanding the role of force as the cause of change in an object's momentum. This understanding culminated with the definition of Newton's three laws of motion, of which the first two explain the role of force and how the lack thereof causes an object to continue in its motion, not stop as was previously believed.

The historical development of the concept of force offers three important lessons, which are in agreement with the p-prim viewpoint:

- it is natural for intuition to assign force the role of maintaining agency,

- once force is assigned the role of maintaining agency, it becomes hard to discern the true role of force,

- it is important to understand the concept of momentum before the role of force can be correctly understood.

\section{Recommendations for Teaching}

As both history and p-prims seem to support the fact that understanding the role of momentum as a maintaining agency is an important step for understanding the concept of force, we offer a few recommendations for teachers that they can implement in their classes:

1. As understanding the role of momentum requires understanding the concept of momentum, we believe that momentum should be introduced before force early in a physics course.

2. To strengthen the connection between momentum, force and their roles in motion, we recommend not using the traditional division of concepts into kinematics and dynamics. Instead we believe momentum can be connected to uniform linear motion, while force can be connected to motion with constant acceleration. In our own mechanics course, we have settled for the following order of teaching the above-mentioned concepts: uniform linear motion, momentum and the conservation of momentum, force and its effects, motion with constant acceleration, combining forces and Newton's laws of motion.

3. After thoroughly analyzing momentum the concept of force should be introduced as the mutual interaction of two objects. Subsequently its role as an actuating agency for motion can be discovered with an activity, where students observe the effects force has on motion. Students release a ball on an inclined plane from a 
fixed height. At the end of the inclined plane students lay different materials on which the ball should move. Then they observe how fast the ball stops moving on different materials, which leads them to observe the effects different friction forces have on motion. After observing that a larger value of friction force leads to the ball stopping faster, they are led to think about and discuss what is needed for the ball to never stop moving. Through this activity students can observe that force changes the momentum of the object, while also can reach the understanding that it is momentum which maintains motion not force.

4. When students have been introduced to combining force and Newton's laws of motion, an important activity for students is finding forces affecting objects in different states of motion. One example of such motion is a car moving with a constant velocity on a flat plane. A typical student response for this example is to draw a net force affecting the car in the direction of motion. The teacher does not dismiss the idea right away but acknowledges the existence of a physical quantity in that direction, and then he shows it to be the momentum of the car not a net force. This way, the teacher reinforces the idea that constant velocity is connected to momentum, or that the maintaining agency of motion is momentum, while force is its actuating agency.

The above-mentioned recommendations have been implemented in three different classes. The first of them presented a preliminary research of the effectiveness of the plan. The class was composed of a group of 20 students 15-17 years old. Using the Force Concept Inventory (FCI) test developed by Halloun and Hestenes (1992) the classroom achieved an average score of $20 \%$ in the pre-test and $69 \%$ in the post-test. We consider this to be a significant gain considering that we focused only on the concept of force in linear motion, which is not the only topic covered by the FCI test.

The two newer classes represented the first stage of a larger research to understand the effects of the plan prepared based on our previous recommendations. An interesting result immerged in students' responses through different activities in those classes. For example, when introducing the concept of centrifugal force, students analyzed forces affecting a rotating object. Most of the students chose to draw momentum in the direction of motion right at the beginning to avoid confusing it with net force. This shows that assigning the role of maintaining agency to momentum can have a significant effect on students understanding of forces. Another interesting effect was when considering motion after centripetal force ceases to act on an object. Students not only correctly observed that the object will continue to move on a straight line, but the explained their hypotheses using the understanding of momentum as a maintaining agency, for example a student's reaction was: "the centripetal force stops acting on the ball after leaving canal, therefore it only has its 
momentum now. The constant momentum will cause the object to continue in its path without changing it".

The FCI test was applied on those two classes as well. The classes consisted of 28 students 15-17 years old. The pre-test achieved a result of $21 \%$ while the post test achieved a result of $73 \%$. We do not consider those results telling as per the small number of students in classes and therefore we point to the qualitative effects of the plan as a better basis for evaluating those effects. However, both the quantitative and qualitative results of the plan will be thoroughly examined throughout the next few years.

\section{Conclusion}

Most physics teachers have met with the student preconception that a force is needed to maintain motion. In our paper we analyzed this preconception through a p-prim point of view, where we saw that the main problem is that students assign the role of maintaining agency to force. To avoid such assignment, we proposed that the role of maintaining agency should be occupied before students are introduced to the concept of force, and that the role of the maintaining agency should be given to momentum. By taking a brief look at the historical development of the concept of force, we saw that a similar problem has occurred in history and needed a similar solution to what we proposed.

Based on the p-prims viewpoint and on the historical parallel, we offered a few recommendations for introducing the concepts of momentum and force. Two of the recommendations were focused on the order of introducing the taught concepts, while two were focused on activities that help emphasize the roles of momentum and force in motion. While the results of preliminary research and the first phase of a broader research were encouraging, the broader research project is still underway to verify the effectiveness of a plan based on our offered recommendations.

\section{Acknowledgments}

This work was supported by the Slovak Research and Development Agency, project Science curriculum 2020 (Grant number APVV-14-0070). 


\section{References}

Baez, J. (2008, January 7). A Tiny Taste of the History of Mechanics. Retrieved August 13, 2018, from The n-Category Café: https://bit.ly/2MbsIPj

diSessa, A. A. (2016). A Friendly Introduction to "Knowledge in Pieces": Modeling Types of Knowledge and Their Roles in Learning. In G. Kaiser, H. Forgasz, M. Graven, A. Kuzniak, E. Simmt, \& B. Xu, Invited Lectures from the 13th International Congress on Mathematical Education (pp. 65-84). Hamburg: Springer Nature.

Dugas, R. (1955). A History of Mechanics. London.

Graney, C. M. (2013). Mass, Speed, Direction: John Buridan's 14th-Century Concept of Momentum. The Physics Teacher, 51, 411-414.

Hammer, D. (1996). Misconceptions of P-Prims: How May Alternative Perspectives of Cognitive Structure Influence Instructional Perceptions and Intentions? The Journal of the Learning Sciences, 5(2), 97-127.

Hecht, E. (2015). Origins of Newton's First Law. The Physics Teacher, 53(2), 80-83.

Hestenes, D., Wells, M., \& Swackhamer, G. (1992). Force Concept Inventory. The Physics Teacher, 30(3), 141-158.

Kortland, K., \& Poorthuis, H. (2017). Handboek natuurkundedidactiek. Epsilon Expenditure.

McDermott, L. C. (1997). Students' Conceptions and Problem Solving in Mechanics. In A. Tiberghien, L. E. Jossem, \& J. Barojas, Connecting Research in Physics Education with Teacher Education (pp. 1-6). International Commission on Physics Education.

Miao, K. X. (2016). Physics, Chapter Two: Concepts of Force in History. Retrieved August 13, 2018, from The Catalyst: https://bit.ly/2OA6GCP

Wildberg, C. (2016, Spring). John Philiponus. Retrieved August 13, 2018, from Stanford Encyclopedia of Philosophy: https://stanford.io/2Bhmnx1 